\section{Buchrezension zu: Gartenvögel rund ums Jahr}

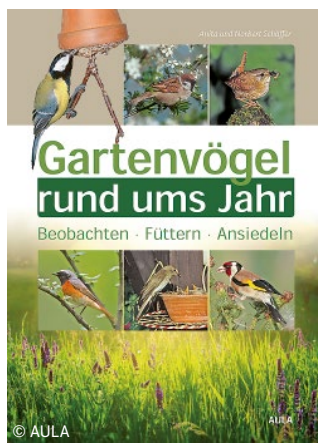

Gartenvögel rund ums Jahr Beobachten - Ansiedeln Füttern

Anita und Norbert Schäffer

300 S., 297 Abb., 3 Karten.

AULA-Verlag, 2020. HC, 14,95 €. ISBN: 9783891048214

DOI: $10.1007 / \mathrm{s} 12268-020-1324-3$ (C) Der Autor 2020

Die intensive Auseinandersetzung mit der Vogelwelt ist ja eine beinahe meditative Beschäftigung, die in erster Linie dazu anhält, stets ganz genau hinzuschauen und hinzuhören. Das günstige Hardcover von Anita und Norbert Schäffer ist für den Einsteiger ein ganz hervorragender Führer, der jede Menge Hintergrundinformationen zum Leben und Lebensraum unserer Stadtund Gartenvögel bietet.

Barcodes zum jeweiligen Gesang haben sich in den aktuellen Vogelbüchern ohnehin durchgesetzt, und die hier vorgestellten 37 Stadtvögel sind sämtlich mit den Tondateien, die Hans-Heiner Bergmann erstellt hat, verlinkt. Wer sich aber wie die Schäffers schon lange mit der Vogelbeobachtung beschäftigt, der kommt nicht umhin, die erschreckende Dezimierung von Individuen und Arten zu registrieren, die im Buch ebenfalls thematisiert wird. Rachel Carson hatte bereits 1962 vor einem „silent spring“, also einem Frühling ohne Vogelstimmen gewarnt - zugehört haben die Wenigsten. Die aufsehenerregende „Krefelder Studie“ von 2017 hat nun gezeigt, dass über einen
Zeitraum von nur 27 Jahren an 60 Beobachtungsstationen in Schutzgebieten die Biomasse an Fluginsekten um $75 \%$ zurückgegangen war. Dies wird hauptsächlich auf den Einsatz von Pestiziden und eine anhaltende Eutrophierung zurückgeführt. Unter dem Einfluss der Landwirtschaft gehen derzeit viele spezialisierte Vogelarten fast gänzlich zurück: Kiebitz (-90\%), Feldlerche (-50\%) und Rebhuhn (-95\%). Schlimmer noch in der Stadt: Hier setzt sich in der Regel eine gute Handvoll Arten durch, die sich eben nicht nur von Insekten ernähren. Nur ein geschultes Auge wird aber in der Stadt ab und zu noch einen Stieglitz, eine Schwanzmeise oder ein Wintergoldhähnchen wahrnehmen. Die gut gemeinte Fütterung zur Brutzeit vergrößert die Probleme sogar noch, weil Jungvögel an dem Kohlenhydratüberschuss schon im Nest zugrunde gehen.

Sicherlich gilt: Gärten können unsere Wälder nicht ersetzen. Wer aber mithelfen möchte, unsere Vögel zu schützen, der sollte sich nicht allein über Nisthilfen, sondern auch über Hauskatzen, Wildwiesen und Brachflächen, über die Anlegung eines Komposthaufens und eine gesunde Mischung autochthoner Pflanzenarten im Garten Gedanken machen. Der reich bebilderte Band aus dem AULA-Verlag liefert dazu eine Menge wertvoller Tipps und Hinweise.

Thomas Floss,

Helmholtz Zentrum München, tfloss@helmholtz-muenchen.de

Funding Open Access funding provided by Projekt DEAL.
Open Access Dieser Artikel wird unter der Creative Commons Namensnennung 4.0 International Lizenz veröffentlicht, welche die Nutzung, Vervielfältigung, Bearbeitung Verbreitung und Wiedergabe in jeglichem Medium und Format erlaubt, sofern Sie den/die ursprünglichen Autor(en) und die Quelle ordnungsgemäß nennen, einen Link zur Creative Commons Lizenz beifügen und angeben, ob Änderungen vorgenommen wurden. Die in diesem Artikel enthaltenen Bilder und sonstiges Drittmaterial unterliegen ebenfalls der genannten Creative Commons Lizenz, sofern sich aus der Abbildungslegende nichts anderes ergibt. Sofern das betreffende Material nicht unter de genannten Creative Commons Lizenz steht und die betreffende Handlung nicht nach gesetzlichen Vorschriften erlaubt ist, ist für die oben aufgeführten Weiterverwendungen des Materials die Einwilligung des jeweiligen Rechteinhabers einzuholen. Weitere Details zur Lizenz entnehmen Sie bitte der Lizenzinformation auf http://creativecommons.org/licenses/ by $/ 4.0 /$ deed.de. 